Article

\title{
Study of Self-Induced Sloshing in Model Test of a Water Retaining Weir
}

\author{
Yulong Hua *, Fulin Cai, Jianxu Zhou and Wen Zhang \\ College of Water Conservancy and Hydropower Engineering, Hohai University, Nanjing 210098, China; \\ flcainj@163.com (F.C.); jianxuzhou@163.com (J.Z.); linketracy@126.com (W.Z.) \\ * Correspondence: damienh@hhu.edu.cn
}

Received: 11 July 2017; Accepted: 25 August 2017; Published: 30 August 2017

\begin{abstract}
This paper presents for the first time observations of a self-induced sloshing phenomenon in the water-retaining weir model. Fast Fourier Transform method is used to detect the dominant frequency for the sloshing water. The characteristics of the sloshing water in terms of sloshing strength and frequency are experimentally studied for six different cases. Results show that both sloshing regions and sloshing strength depends on not only the water levels but also the inlet velocities, and the relationship between them is displayed in this paper. Different prediction models for sloshing frequencies are built and compared with the experimental results, with discussions of the differences between them. For higher water level sloshing region, a new factor is introduced to the Faltinsen's prediction formula, leading to a better agreement between prediction and experimental results. The mechanism of the self-induced sloshing is also discussed in this paper.
\end{abstract}

Keywords: self-induced sloshing; water-retaining weir; fast fourier transform; stepped low-pressure water supply

\section{Introduction}

The hydraulic characteristics of a water-retaining weir structure have been investigated in the past decade due to its practical function in the water control project. For instance, a weir has been built to connect two pressurized pipes from a higher position to a lower one, which is one of the key structures in the Tianjin section of the Middle Route Project (MRP) for South-to-North Water Transfer, China. By delivering water from the south to the north, the construction of the MRP has effectively alleviated extreme water shortages in northern China [1]. The function of the weir is twofold. One is to adjust water level distribution automatically in the pressurized pipe; the other is to guarantee that the pipes are full of water for all steady conditions.

Previous experimental studies focused on three flow patterns with regard to the water-retaining weir, i.e., free overfall flow, submerged overfall flow, and transition flow [2]. Especially for the overfall flow condition, the changes of the flow coefficient with the weir head was studied in a model test [3].

A self-induced oscillation of a free surface was observed in a thin rectangular tank with a horizontally injected plane jet. The sloshing grows in a certain region of the flow rate and the water level, and the frequency is measured to be the same as the 1st-order eigenvalue of the surface oscillation in the tank [4]. With a much lower water level, for the same structure, another self-induced oscillation caused by a plunging jet was discovered and studied [5]. Connecting the same structure to a large downstream tank, self-induced oscillation was observed to be the same of the liquid oscillation in a U-tube [6]. Self-induced oscillation was also captured in the rectangular tank, with a vertical plane jet at the bottom [7]. Two self-induced oscillations with their natural frequency were discovered in a cylindrical tank [8]. After a review of the characteristics of these self-induced oscillations, the mechanisms of the oscillations were investigated [9]. A new type of self-induced free-surface 
oscillation was discovered in a cylindrical tank, from the bottom centre of which an upward round jet was injected into and it impinged on the bottom of an upper-inner-structure in a Liquid Metal Fast Breeder Reactor (LMFBR) nuclear reactor [10]. In the same tank without the upper-inner-structure, self-induced sloshing was observed when an upward round jet on the tank axis created a surface swell at the impingement point. Jet behavior was investigated both experimentally and numerically [11]. Self-excited sloshing was observed in the cooling circuit of the French faster breeder reactor (FBR). Two analytical models were proposed to describe the sloshing [12,13].

Among all the experimental studies of the water-retaining weir in MRP [2,3,14], a more complicated flow field has been observed in the transition flow, which is often characterized with waves in the free surface and termed as self-induced sloshing or self-induced oscillation(shown in Figure 1).Within a period, water surface cycles from moment $t_{1}$ to $t_{4}$. This phenomenon is of great importance in both theoretical and practical hydraulic engineering. However, the study of this phenomenon as well as its mechanism is limited. Our present model test aims to observe the self-induced sloshing in a water-retaining weir under different flow rates and water levels. The mechanism behind this phenomenon is discussed.

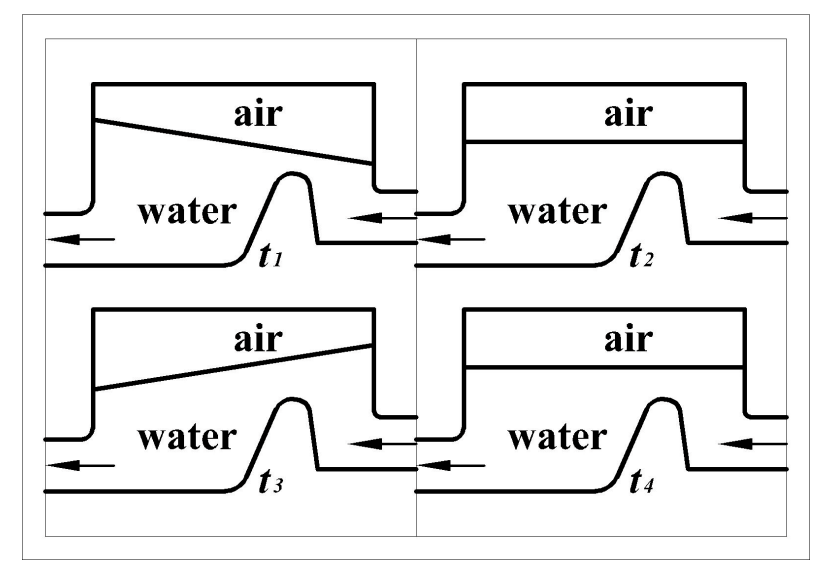

Figure 1. Sloshing in the water-retaining tank.

\section{Experiment Setup \&Methodology}

\subsection{Experiment Setup}

A schematic view of a simplified water-retaining weir tank is shown in Figure 2a. Water comes into the tank from the right side, passes over the weir and flow out from the left side. Figure $2 b$ demonstrates the details of the weir applied in the present model test. The length of both inlet and outlet duct is about $5.0 \mathrm{~m}$, which is more than five times the inlet hydraulic diameter. Figure $2 \mathrm{~d}$ is the photo of the weir tank model.

Figure 2c shows the schematic diagram of the flow loop system used in the present study. Water was pumped up from the lower tank to the head tank, from which the inlet flow was supplied to water tank 1. With tank 1, water level was maintained constant before flow entering the water-retaining weir. At the water-retaining weir area, a valve tank 1 was used to control the flow rate and the water level was adjusted by changing the opening of the tail gate after tank 2 . An overflow weir was placed after tank 2 to evaluate the flow rate in the system. All structures were firmly fixed to the floor, and no resonant fluid oscillation induced by structural vibration was observed.

Six different flow rates were applied in the experiments, which were categorized as case 1 to case 6 and details are listed in Table $1 . V$ represents the average velocity at the inlet section of the pipe. For each case, the water head above the weir $H_{1}$ is a constant value when the flow pattern is in free overfall flow; for this flow pattern, the average velocity for water above the weir is described as $V_{1}$. For different $H_{1}, V_{1}$ can be calculated by $Q / H_{1} / a$, where $Q$ is the inlet discharge, and $a(=22 \mathrm{~cm})$ is the 
distance between the front and back walls in the present model test. Experiments were conducted by gradually increasing water level for each case in order to achieve different water level conditions. The time interval between two water levels is set to be $10 \mathrm{~min}$ to ensure enough transition time for the flow so that the residual effect is minimized. The water depth used in the present study is the average value measured by a liquid level sensor.

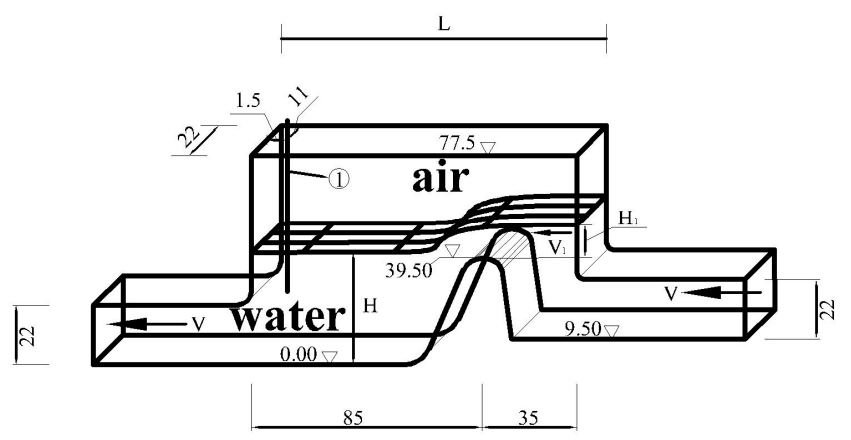

(a)

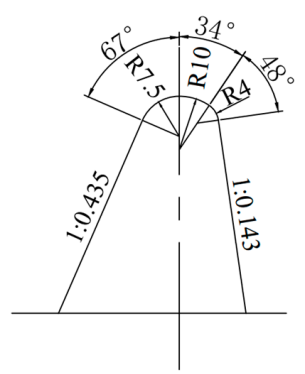

(b)

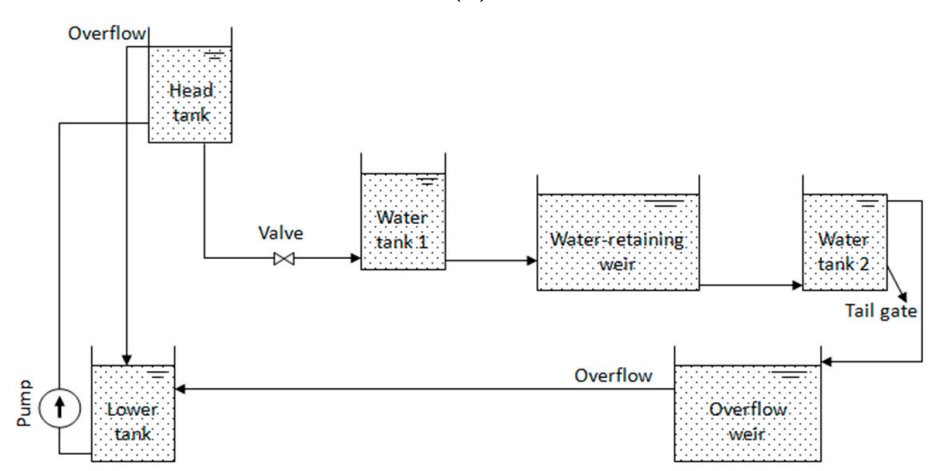

(c)

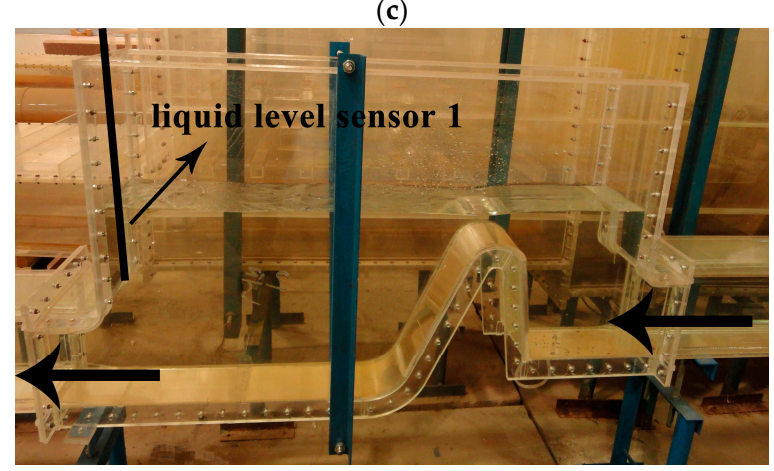

(d)

Figure 2. Details of the test tank and the experiment test system: (a) Detail drawing of the test tank; (b) Detail drawing of the weir (dimensions in $\mathrm{cm}$ ); (c) Schematic diagram of the test loop (dimensions in $\mathrm{cm}$ ); (d) Photo of the experimental model of the test tank. 
Table 1. Six different cases for model test.

\begin{tabular}{cccc}
\hline Case & $\boldsymbol{V}(\mathbf{m} / \mathbf{s})$ & $\boldsymbol{H}_{\mathbf{1}}(\mathbf{c m})$ & $\boldsymbol{V}_{\mathbf{1}}(\mathbf{m} / \mathbf{s})$ \\
\hline 1 & 0.033 & 2.9 & 0.252 \\
2 & 0.074 & 4.5 & 0.360 \\
3 & 0.119 & 5.9 & 0.445 \\
4 & 0.158 & 7.0 & 0.497 \\
5 & 0.198 & 7.9 & 0.551 \\
6 & 0.239 & 8.7 & 0.606 \\
\hline
\end{tabular}

\subsection{Methodology}

\subsubsection{FFT Analysis on Autocorrelation Function of Water Level}

The time series of water level at the left vertical wall (shown in Figure 2a) was measured and recorded by the liquid level sensor (1) with a sampling frequency of $50 \mathrm{~Hz}$. The frequency analysis is based on Blackman Tukey Method [15]. It is realized by performing Fast Fourier Transform (FFT) to the autocorrelation function of the water level signals (dimensionless signals in this article). The unbiased form is used in the autocorrelation function. The spectrum with energy and frequencies of the water level signals was obtained through FFT. The energy from the spectrum reflects sloshing amplitude, i.e., the higher value of the energy is, the larger the sloshing amplitude is. Hereafter the sloshing strength discussed in this paper is interpreted by the energy from the spectrum.

\subsubsection{Prediction Formulation for Theoretical Sloshing Frequencies}

\section{2-D Rectangular Vessel Model}

Consider a rectangular vessel with water of depth $H$ and width $L$, shown in Figure 3. The theoretical natural frequency of nth mode sloshing without circulation flow is expressed as follows [16]:

$$
f=\frac{1}{2 \pi} \sqrt{g k \tanh k H}
$$

where $k=n \pi / L$ is the wave number, $n=1,2$, denotes the mode of sloshing, $L$ is the width of the rectangular vessel, $H$ is the water depth of the vessel, and $g$ is gravitational acceleration.

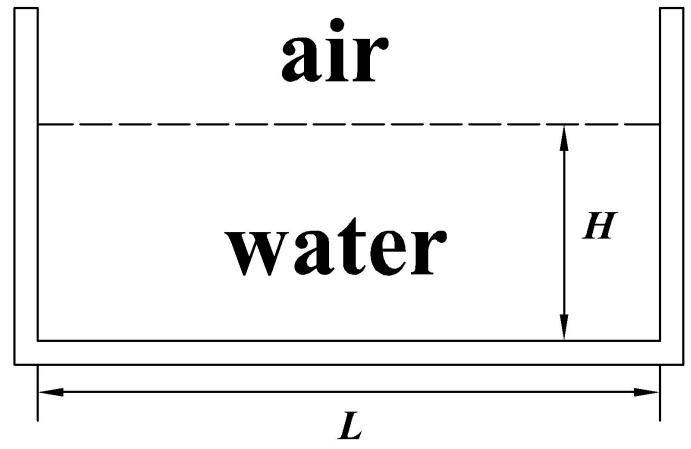

Figure 3. Rectangular vessel with water.

The shape of the water-retaining weir should be simplified as a rectangular one, since Equation (1) is used for the natural frequency of a two-dimensional rectangular water tank.

Equation (1) presents sloshing without surface water velocity.

However, the surface water velocity cannot be neglected for a weir tank system with flow entering and exiting. Therefore, a factor to reflect the effect of surface velocity should be included in the formula, which was discussed and concluded by Hara, F. 1990 [17] as below: 


$$
f=\frac{1}{2 \pi} \sqrt{g k \tanh k H-U^{2} k^{2}}
$$

where $U$ is the velocity of the surface water, and $k, H, g$ represent the same variables in Equation (1).

Rectangular Vessel with Vertical Baffle Model

When water surface downstream of the tank is much lower than the upstream, the model of sloshing near the downstream water could be simplified as water sloshing in a rectangular vessel. With the increase of water level, the downstream and upstream water gradually reaches the same level and the sloshing happens in the whole tank. For this situation, the weir itself should be considered a vertical baffle which cannot be ignored.

Firouz-Abadi [18] provided a diagram summarizing the dimensionless sloshing frequencies as a function of the baffle height in a rectangular tank based on a model test with the baffle located in the middle of the tank, shown in Figure 4. In this diagram, the weir is regarded as a vertical baffle, and the natural frequencies could be predicted.

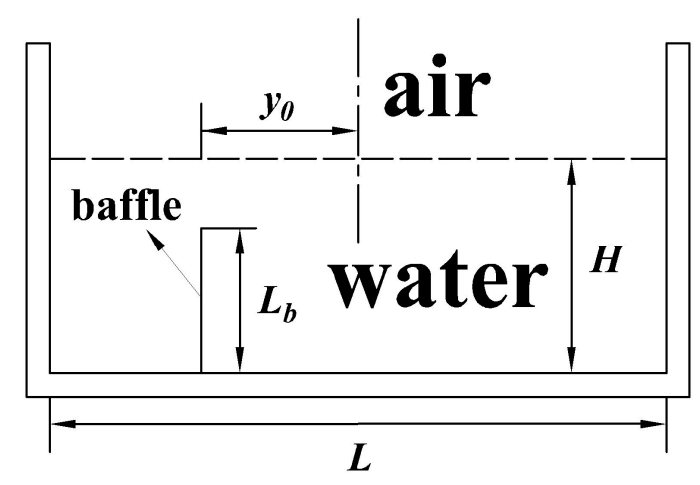

Figure 4. Rectangular vessel with vertical baffle inside.

However, the weir is not located exactly in the middle of the tank. When the effect of a bottom-mounted vertical baffle on two-dimensional sloshing in a rectangular tank is taken into consideration, in Faltinsen's book [19], the frequency $f^{\prime}$ is expressed as,

$$
\frac{f^{\prime 2}}{f^{2}}=1-\frac{2 \pi^{2} n \sin ^{2}\left(\pi n\left(y_{0}+\frac{1}{2} L\right) / L\right)}{\sinh (2 \pi n H / L)}\left(\frac{L_{b}}{L}\right)^{2}
$$

where $f$ is the frequency in a rectangular vessel without baffle, which can be calculated by Equation (2); $y_{0}$ is the displacement of the baffle from the middle of the tank; $L$ is the width of the tank; $L_{b}$ is the height of the baffle; $H$ is the water depth; and $m$ denotes the mode of sloshing.

\section{Results}

\subsection{Self-Induced Sloshing Phenomenon}

With a certain inlet discharge for the whole test system controlled by valve, and a certain water level fixed in water tank 2 , self-induced sloshing phenomenon is observed, and results are shown in Figure 5. There are two water surface sloshing regions. Region 1 is the lower one between water level 1 (the lower boundary water level) and water level 2 (the upper boundary water level). Obvious sloshing phenomenon is observed in this region in front of the weir. The other surface sloshing region is region 2. In this region, water sloshing happens in the whole water-retaining tank. The lower boundary water level of region 2 is water level 3 and the upper one is water level 4.The sloshing phenomenon only happens for water levels within region 1 and region 2, and it disappears outside 
region 1 and 2. For a certain inlet velocity, in any sloshing region, sloshing strength varies with various water levels. For different inlet velocities, definitions of region 1 and region 2 are also different.

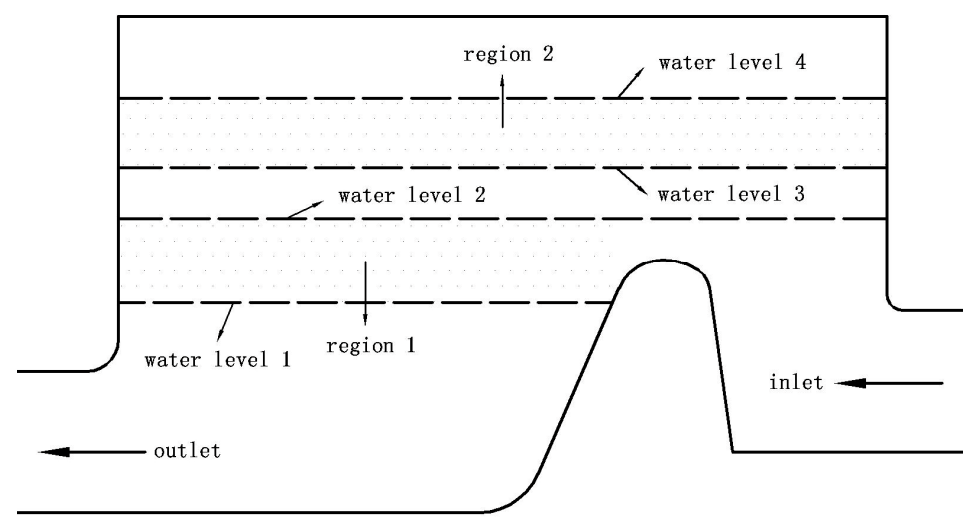

Figure 5. Free surface sloshing regions for self-induced sloshing.

\subsubsection{Relationship between Water Surface Sloshing Region and Water Level}

The water level signals (shown in Figure 6a)are determined by dimensionless waterlevel divided by the weir crest altitude $39.5 \mathrm{~cm}$, and the dimensionless water level is described by variable $h(h=H / 39.5)$. The FFT results of the autocorrelation of signals from 2 different water levels for Case 3 $\left(V_{1}=0.119 \mathrm{~m} / \mathrm{s}\right)$ are displayed in Figure $6 \mathrm{~b}$ as examples. Statistics for all water levels investigated in the present model test are listed in Table 2. For Case 3, in sloshing region 1, the 1st mode sloshing strength increases significantly with water level $h$ increasing from 0.942 to 0.962 , which is reflected by the spectrum energy varying from $0.004 \times 10^{-3}$ to $3.187 \times 10^{-3}$. The sloshing strength starts to decrease gradually when $h$ is higher than 0.962 . Until $h=1.106$ or higher, there is no sloshing phenomenon observed. While for region 2, similar variation of the sloshing strength with the water level is observed. For region 2, level 3 is 1.162 and level 4 is 1.301 . With the increase of water level, the sloshing strength increases and reaches the peak value (corresponding to spectrum of $0.217 \times 10^{-3}$ ) at water level of 1.253. After that, the sloshing strength keeps decreasing to 0 , and to a water level of 1.301. Obviously the water sloshing strength is stronger in region 1 than in region 2. For this inlet velocity $\left(V_{1}=0.119 \mathrm{~m} / \mathrm{s}\right)$, add $H_{1}=5.9 \mathrm{~cm}$ to the altitude of the crest $39.5 \mathrm{~cm}$, be divided by $39.5 \mathrm{~cm}$, and the answer is 1.149. Both the lower water level section (0.942-1.106) and higher one (1.162-1.301) is around the value 1.149, which is the water level upstream the weir for free overfall flow. For all the other cases with different inlet velocities, the feature of the sloshing amplitude with the water level is similar to Case 3.

Table 2. Power spectral density with water level (exp.) for case 3.

\begin{tabular}{cccccc}
\hline Region 1 & \multicolumn{2}{c}{ 1st Mode } & Region 2 & \multicolumn{2}{c}{ 1st Mode } \\
\hline $\boldsymbol{H}^{\mathbf{1}}$ & Frequency $\mathbf{( H z )}$ & Spectrum $\left(\times \mathbf{1 0}^{-\mathbf{3}}\right)$ & $\boldsymbol{H}^{\mathbf{1}}$ & Frequency (Hz) & Spectrum $\left(\times \mathbf{1 0}^{\mathbf{- 3}}\right)$ \\
\hline 0.942 & 0.94 & 0.004 & 1.162 & 0.6 & 0 \\
0.954 & 0.94 & 0.026 & 1.175 & 0.57 & 0.025 \\
0.962 & 0.9 & 3.187 & 1.2 & 0.57 & 0.113 \\
0.975 & 0.9 & 2.512 & 1.228 & 0.58 & 0.158 \\
1 & 0.88 & 1.467 & 1.246 & 0.58 & 0.189 \\
1.061 & 0.85 & 0.194 & 1.253 & 0.58 & 0.217 \\
1.094 & 1.17 & 0.057 & 1.271 & 0.59 & 0.128 \\
1.106 & 0 & 0 & 1.286 & 0.59 & 0.028 \\
- & - & - & 1.301 & 0.6 & 0 \\
\hline
\end{tabular}




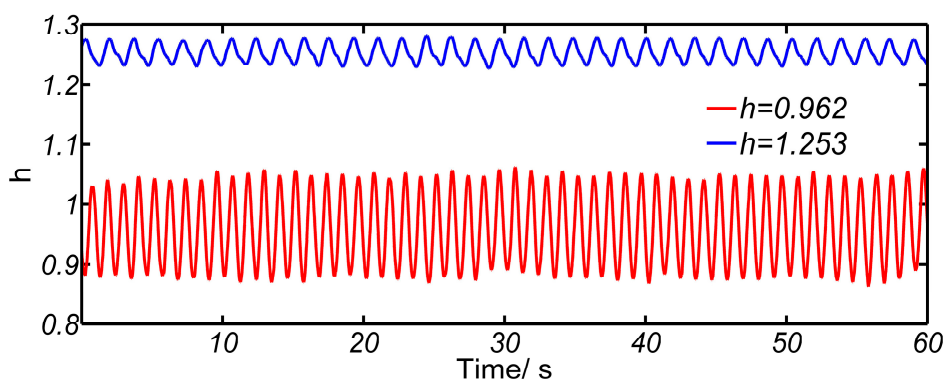

(a)

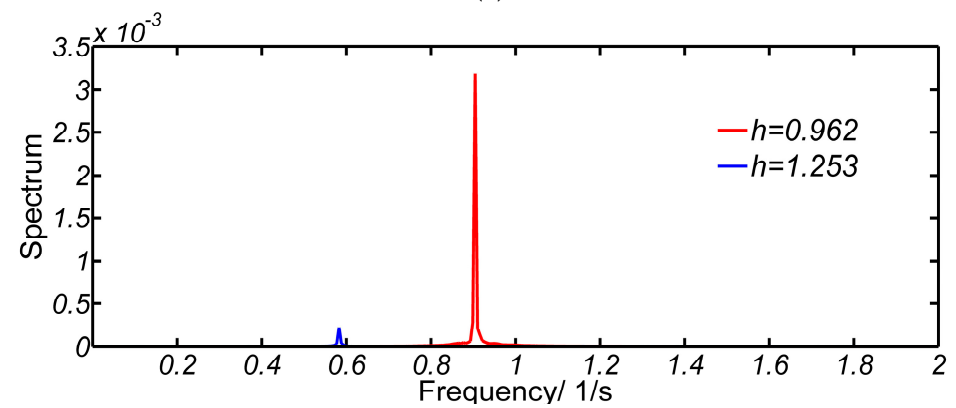

(b)

Figure 6. (a) Water level oscillation for two different water levels, $h=0.962$ and $h=1.253$ in Case 3; (b) Frequency analysis for two different water level signals, $h=0.962$ and $h=1.253$ in Case 3.

\subsubsection{Relationship between Water Surface Sloshing Regions and Inlet Velocity}

Six different inlet velocities varying from $0.033 \mathrm{~m} / \mathrm{s}$ to $0.239 \mathrm{~m} / \mathrm{s}$ have been applied in this experiment. Water surface sloshing regions exists for all the six cases, but with different features as shown in Figure 7.

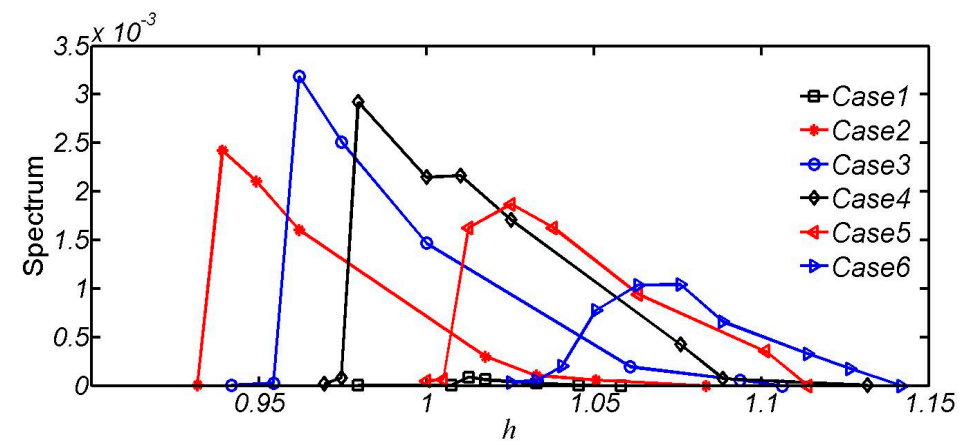

(a)

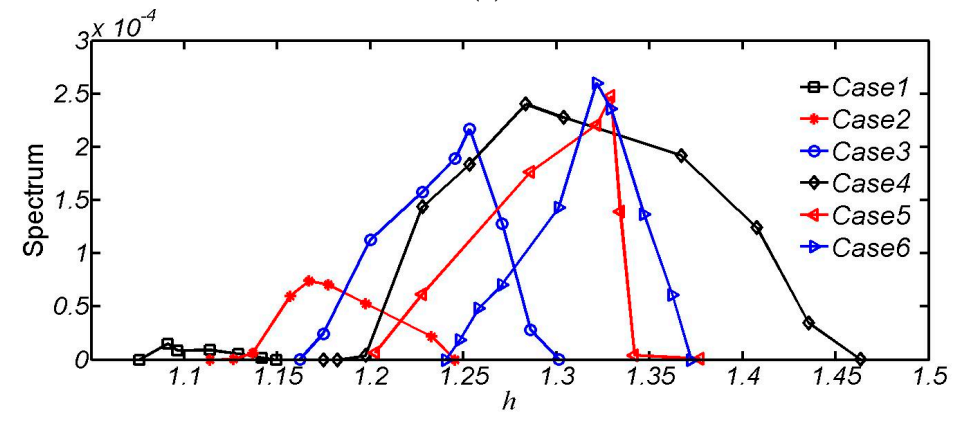

(b)

Figure 7. (a) Sloshing strength vs. water levels (in region 1); (b) Sloshing strength vs. water levels (in region 2). 
With the increase of the inlet velocity, the highest sloshing strength increases for Case 1 to 3 and begins to decrease for Case 4 to 6 in region 1. However, the average water level of the sloshing region goes up with the inlet velocity as shown in Figure 7a, except from Case 1 to Case 2 the average water level demonstrates a decrease. Water level associated with the strongest sloshing strength for each inlet velocity also increase with the increase of inlet velocity (except from Case 1 to Case 2).

For region 2, as shown in Figure 7b, the highest sloshing strength increases quickly from Case 1 to Case 3 and gradually reaches the peak value for Case 6. Actually, there is not much difference in the highest sloshing strength among Cases 4-6. At the same time, the ranges of sloshing water levels between level 3 and level 4 becomes bigger first with the increase of velocities till Case 4 , and then becomes smaller for Case 5 and Case 6. But the water level associated with the strongest sloshing strength increase all the way with the increase of the velocity. For the highest two velocities, the water levels are almost the same.

\subsection{Sloshing Frequencies Results from the Prediction Formula}

\subsubsection{With/without Consideration of Surface Water Velocity (for Region 1)}

When the sloshing phenomenon happens in region 1 (Figure 5), the downstream part of the tank can be simplified as a rectangular vessel. Since the downstream wall of the weir is a wall with slope, the width of the simplified rectangular vessel varies from $65 \mathrm{~cm}$ to $78 \mathrm{~cm}$. The 1 st node sloshing frequency calculated by Equation (1) is shown in Figure 8a (shown by Rec. which is short for rectangular vessel model) with water level h changing from 0.85 to 1.15 .

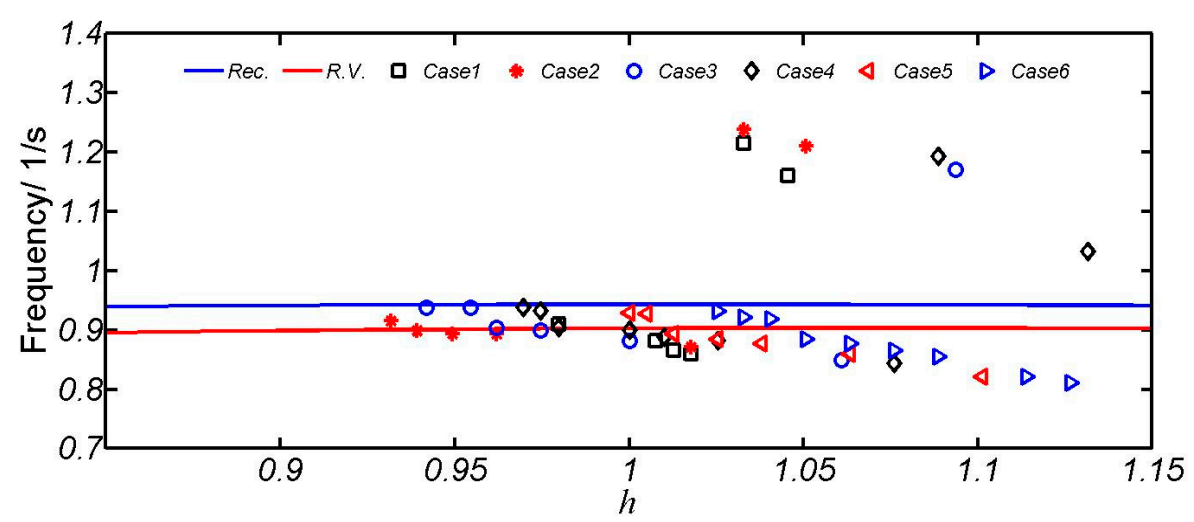

(a)

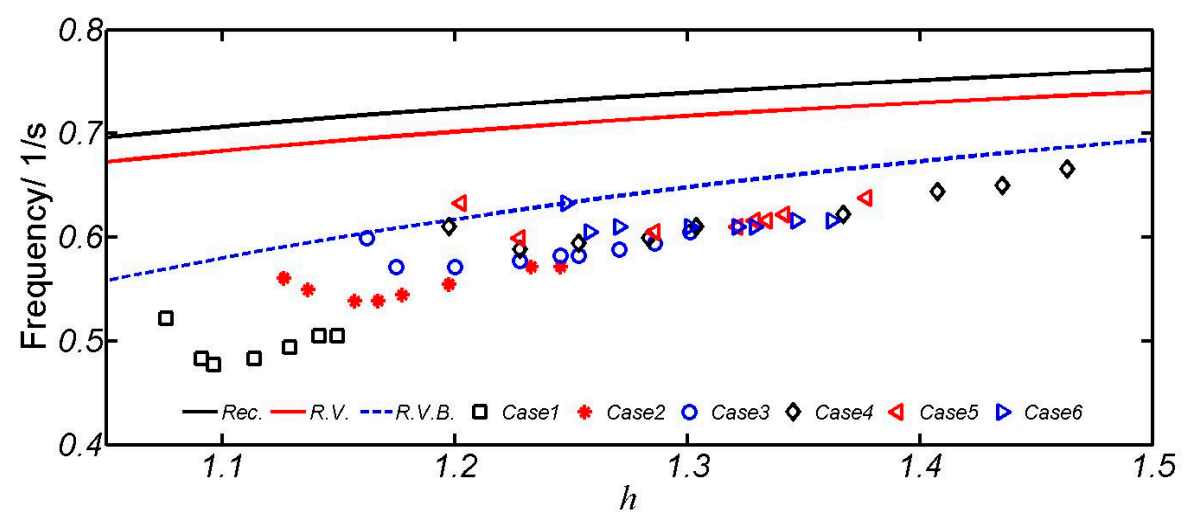

(b)

Figure 8. (a) Oscillating frequencies vs. water levels in the tank (for region 1); (b) Oscillating frequencies vs. water levels in the tank (for region 2). 
In fact, Equation (1) is only for sloshing water in the tank without consideration of surface water velocity and the stream line of flow under this condition is draw in Figure 9a, according to the flow pattern of free overfall flow described in Xu Guofeng's Master Thesis [14]. However, Equation (2) should be used here with the existence of free surface velocity. Since the free surface water flows from the right to the left, and flows down with water coming down above the weir, the downstream surface velocity should be the same as the surface water velocity of water above the weir injecting to the downstream. Therefore the water velocity above the weir $V_{1}$ (listed in Table 1 ) is used in the present paper instead of the downstream surface water velocity. For different cases, the average of $V_{1}$ in Case 1 (the smallest $V_{1}$ ) and Case 6 (the biggest $V_{1}$ ) is used to predict the result (shown by R.V. short for Rectangular vessel considering surface water Velocity) by Equation (2).

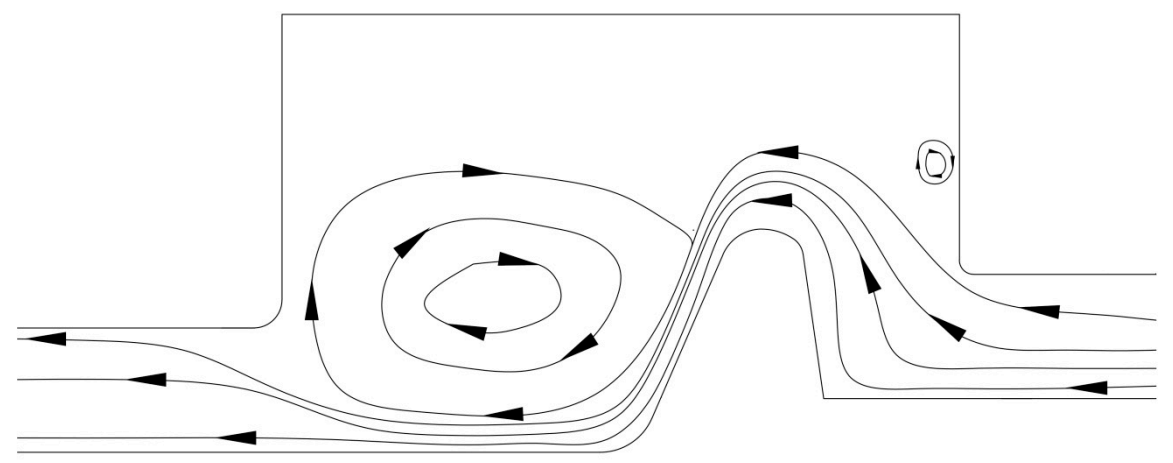

(a)

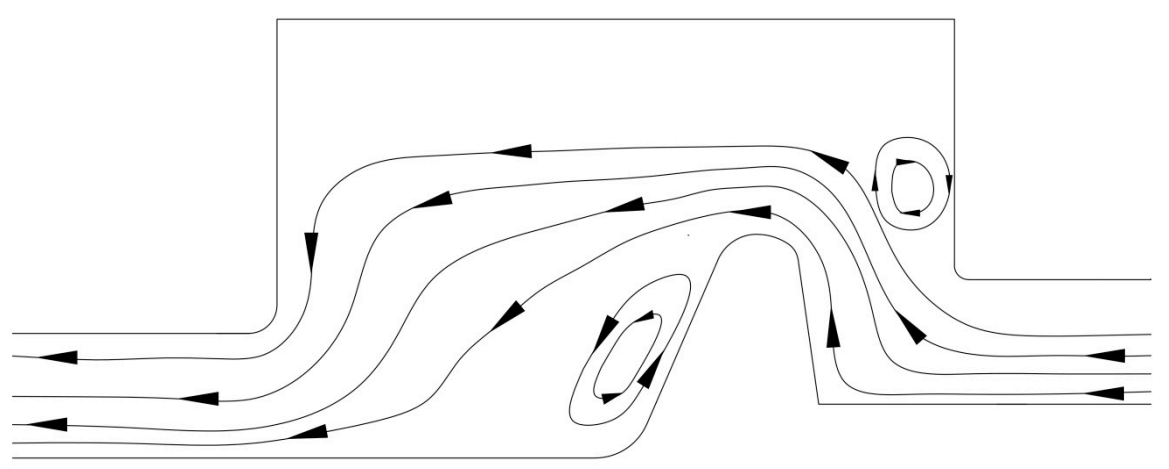

(b)

Figure 9. Streamline for two sloshing regions. (a) For region 1; (b) For region 2.

\subsubsection{With/without Consideration of Baffle Position (for Region 2)}

For the tank with higher water levels, sloshing happens in region 2. With the existence of the weir, the whole tank cannot be simplified as a rectangular vessel anymore. The weir acts as a vertical baffle and as a resistance for the sloshing water.

The diagram of dimensionless sloshing frequency against the baffle height in a rectangular tank is displayed in [18]. This is based on a model test on a baffle located in the middle of the tank and the 1st mode natural frequencies could be calculated in this way. In that paper, the baffle is located in the middle of the tank. To use this curve, the weir is taken as a baffle in the middle of the test tank.

However, the weir is not located exactly in the middle of the tank, so a formula considering the exact position of the weir is required to provide more accurate information. In Faltinsen's Equation (3), $y_{0}$ represents the horizon distance between the vertical baffle and axis of the vessel. The streamline of the flow under this situation is shown in Figure $9 \mathrm{~b}$ with corresponding free surface water velocities $V_{1}$ presented in Table 1. In Equation (3), $f$ is the frequency from Equation (2), with consideration of 
water surface velocity. So the sloshing frequencies predicted by Equation (3) is displayed in Figure 7a (shown by R.V.B short for Rectangular vessel considering surface water Velocity with a Baffle in the middle) with consideration of both surface velocity and the weir position.

\section{Discussion}

\subsection{Mechanism for Self induced Sloshing}

Although the frequencies by prediction formula are close to the experiment results for region 1 , some differences should be identified. Specifically, the frequencies calculated by predication formula increases lightly with the increasing water levels while the frequencies from experiments decrease with the increasing water levels as shown in Figure 8b. The difference results from the simplified prediction model, of which the downstream tank is not rigidly a rectangular tank. Its upstream wall is not vertical but sloped. Therefore, the range of free surface water is larger with the increase of water level, and the frequency will be smaller than that of the rectangular one at the same water level. However, for the physical water tank, the upstream wall is not straight. Since the top of the wall is the downstream part of the weir with curved figure. That means that with the same increased water levels, free surface water length increases more, and by Equation (1), the frequencies decrease with the larger $L$.

With this law, the sloshing frequency decreases with the increase of water level. While for the highest water levels in region 1, the sloshing frequency actually shows a sudden significant increase. As discussed previously, the factor affecting the sloshing frequency includes not only the size of the tank and the water level, but also the free surface water velocity. According to Equation (2), the free surface water velocity shows a slight decrease when water level increase. Besides, when water level is higher than the altitude of the weir crest, the thickness of water into the downstream tank gets bigger. This will lead to the equivalent $L$ of water in the downstream tank much smaller and sudden increase of the sloshing frequency.

Meanwhile, the flow pattern begins to change when water level reaches a high value. From Figure 9a,b, the flow pattern transforms from free overfall flow to submerged overfall flow. From one stable flow pattern state to another, the path of the main flow will be unstable and vortex may appear, which results in the self-induced sloshing [20]. For the two prediction formulas, results from Equation (2) are much closer to experiments results than that from Equation (1) considering the effect of the free surface water velocity.

While for region 2, with the consideration of the baffle position, and surface water velocity, the prediction results (R.V.B.) are closer but slightly bigger than the experimental results (shown by Figure $8 b$.

\subsection{New Formulawith Consideration of the Width of the Vertical Baffle in Region 2}

For the simplification of the weir as a thin baffle, the height is considered rather than its thickness (B). Also, that formula would ignore part of the resistance caused by the weir. Since the thickness of the baffle plays the same role as the height of the baffle, baffle thickness and its weighting factor $e$ for resistance could be introduced to Equation (3) as

$$
\frac{f^{\prime 2}}{f^{2}}=1-\frac{2 \pi^{2} n \sin ^{2}\left(\pi n\left(y_{0}+\frac{1}{2} L\right) / L\right)}{\sinh (2 \pi n h / L)}\left(e B+\frac{L_{b}}{L}\right)^{2}
$$

where the average width of the baffle $(B)$, or the baffle thickness is 0.214 in this present experiment.

For inlet velocity of $0.119 \mathrm{~m} / \mathrm{s}$ (Case 3), value of $e$ is calculated as 0.4 . And for the other five cases, results from Equation (4) with the same value of $e$ and B, are displayed in Figure 10a-f (F.B.b. short for Formula for vessel with Baffle considering its thickness).All the predictions by Equation (4) are much closer to the experimental results in region 2. 


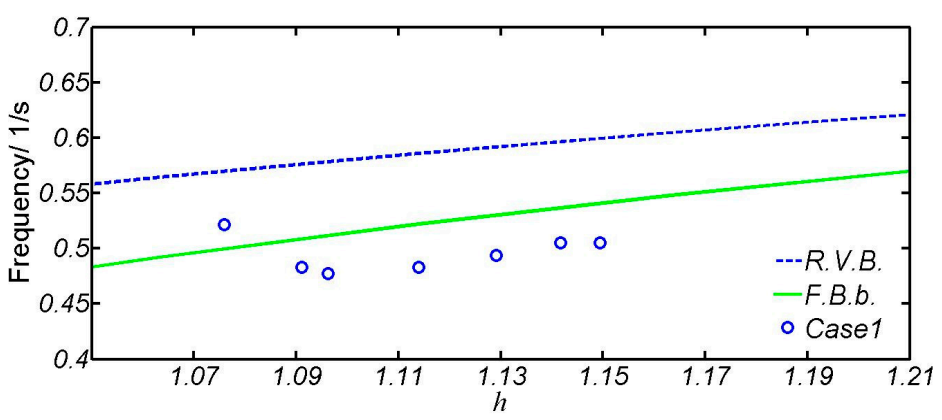

(a)

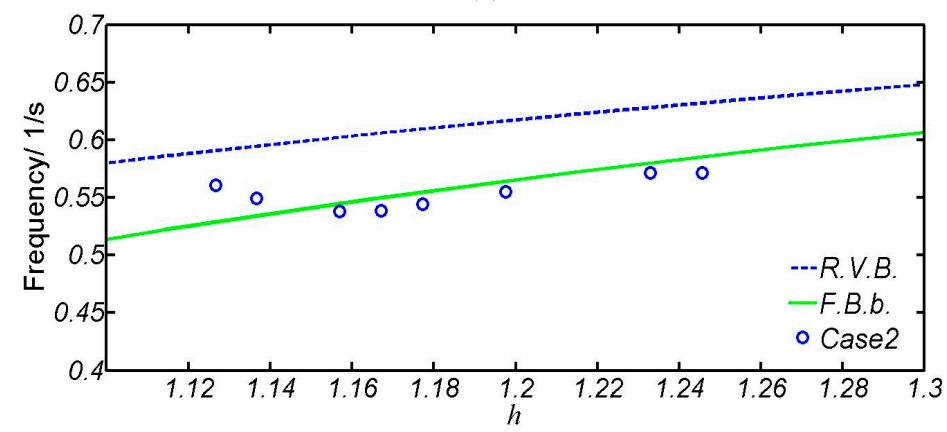

(b)

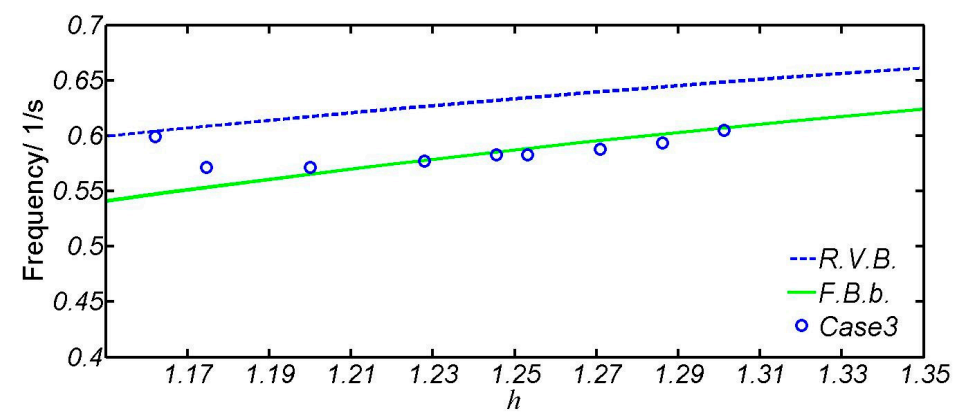

(c)

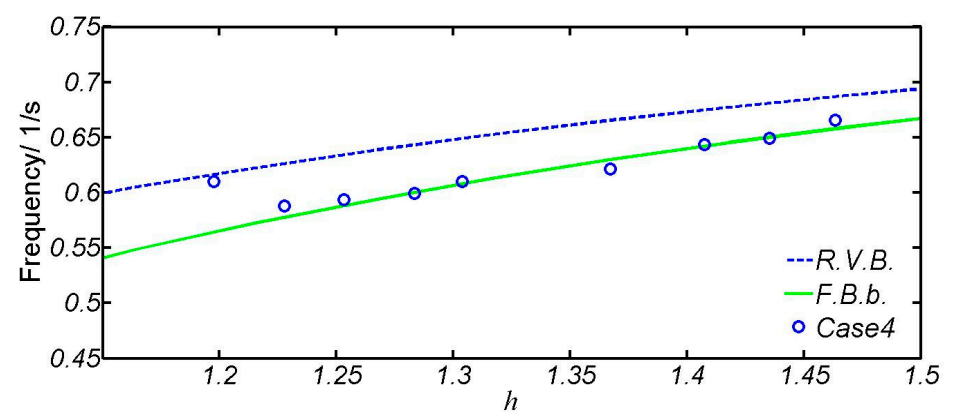

(d)

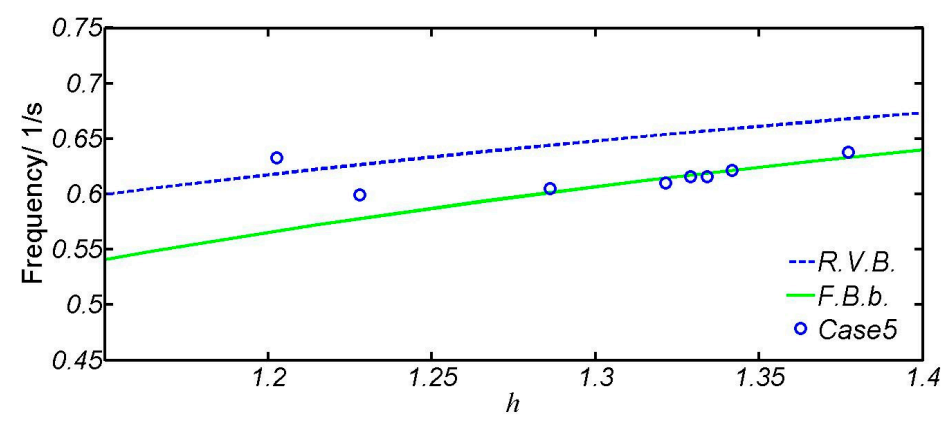

(e)

Figure 10. Cont. 


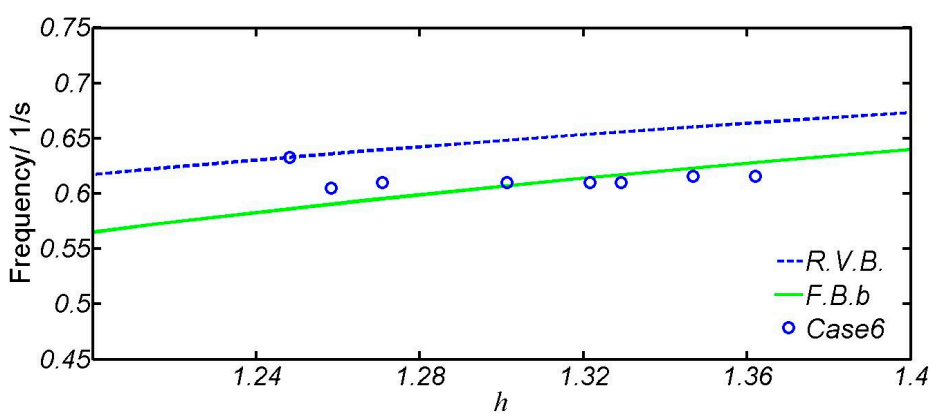

(f)

Figure 10. Prediction results with consideration of the free surface velocity and width of the weir. (a) Case 1; (b) Case 2; (c) Case 3; (d) Case 4; (e) Case 5; (f) Case 6.

However, for the lower water levels in sloshing region 2, the difference is slightly bigger. At low water levels, sloshing pattern is going to change according to Figure 9a,b. Also, the frequency for sloshing in region 1 is much higher than that in region 2. So when water level is low enough, the sloshing frequency gets larger than others of high water levels.

\section{Conclusions}

Self-induced sloshing phenomenon in the water tank with a water-retaining weir is experimentally studied. Both the sloshing strength and frequency are investigated with FFT method. For any inlet velocity tested in the experiment $(V=0.033,0.074,0.119,0.158,0.198,0.239)$, obvious self-induced sloshing phenomenon only exists in water surfaces within two small sloshing regions around the weir crest. The sloshing region varies with the inlet velocity, and the sloshing strength varies with water levels in the two sloshing regions. For the two sloshing regions, the sloshing models are simplified as rectangular vessel and rectangular vessel with a vertical baffle. Frequencies predicted from those two models are close to the experiments especially for the sloshing region with lower water levels. A new factor is introduced to the formula of the simplified weir tank model for the sloshing region with higher water levels, and the prediction frequencies are closer to the frequencies in experiments.

Future work includes further study on the mechanism of the self-induced sloshing, and other hydraulic phenomenon such as air entrainment with the CFD method.

Acknowledgments: The author gratefully acknowledge all the help from Yuedong Zhu and all the other researchers in the Lab (Research Institute of Hydraulic and Hydropower Engineering), and the support by the National Natural Science Foundation of China [grant No. 51079051].

Author Contributions: Yulong Hua conceived this paper, designed and performed the experiments, and analyzed the data; Fulin Cai and Jianxu Zhou assisted the experiment and revised the paper; Wen Zhang revised the paper and provided some valuable suggestions.

Conflicts of Interest: The authors declare no conflict of interest.

\section{References}

1. Fang, S.; Wei, J.; Wu, B.; Shang, Y. Simulation of transport channel in China's middle route south-to-north water transfer project. J. Tsinghua Sci. Technol. 2009, 14, 367-377. [CrossRef]

2. Yang, M.; Xu, G. Study on hydraulic characteristics of water conservation weir in the south-to-north water transfer project. J. China Rural Water Hydropower 2006, 48, 95-98.

3. Wang, K.; Guo, B. Study on the conveyance capacity of the water-keeping weir. J. Water Conserv. Sci. Technol. Econ. 2010, 16, 1026-1028.

4. Okamoto, K.; Haruki, M.; Tsuyoshi, H. Self-induced oscillation of free-surface in a tank with circulating flow. J. Trans. Jpn. Soc. Mech. Eng. Ser. C 1991, 57, 647-653. [CrossRef]

5. Baba, M.; Madarame, H.; Okamoto, K. Self-induced sloshing caused by plunging jet. J. Trans. Jpn. Soc. Mech. Eng. Ser. B 1999, 65, 2278-2285. [CrossRef] 
6. Okamoto, K.; Madarame, H.; Hagiwara, T. Self-induced manometer oscillation of free surface caused by circulating flow. J. JSME Int. J. Ser. C 1993, 36, 171-176. [CrossRef]

7. Fukaya, M.; Madarame, H.; Koji, O. Growth mechanism of self-induced sloshing caused by jet in rectangular tank (1st self-induced sloshing caused by vertical plane jet). J. Trans. Jpn. Soc. Mech. Eng. Ser. B 1996, 62, 541-548. [CrossRef]

8. Someya, S.; Madarame, H.; Okamoto, K. Self-induced sloshing caused by upward jet impinging on upper-inner-structure. J. Trans. Jpn. Soc. Mech. Eng. Ser. B 1996, 62, 533-540. [CrossRef]

9. Okamoto, K.; Madarame, H. Fluid dynamics of a free surface in liquid metal fast breeder reactors. J. Prog. Nucl. Energy 1998, 32, 195-207. [CrossRef]

10. Someya, S.; Okamoto, K.; Madarame, H. The self-induced free-surface "swell flapping" caused by the interaction among a jet, a free surface and a structure. J. Fluid Struct. 2000, 14, 511-528. [CrossRef]

11. Madarame, H.; Okamoto, K.; Iida, M. Self-induced sloshing caused by an upward round jet impinging on the free surface. J. Fluid Struct. 2002, 16, 417-433. [CrossRef]

12. Kaneko, S.; Nagakura, H.; Nakano, R. Analytical model for self-excited vibration of an overflow flexible plate weir. J. Press. Vessel Technol. 1999, 121, 296-303. [CrossRef]

13. Nagakura, H.; Kaneko, S. Self-Excited sloshing due to the fluid discharge over a flexible plate weir. J. Press. Vessel Technol. 1999, 122, 192-197. [CrossRef]

14. $\mathrm{Xu}, \mathrm{G}$. Studied on Hydraulic Characteristics and Body Type Optimization of Water Conservation Weir. Master's Thesis, Tianjin University, Tianjin, China, 1 December 2006.

15. Blackman, R.B.; Tukey, J.W. The measurement of power spectra from the point of view of communications engineering-Part 1. J. Bell Syst. Tech. J. 1958, 37, 185-282. [CrossRef]

16. Kaneko, S.; Nakamura, T.; Inada, F.; Kato, M.; Ishihara, K.; Nishihara, T.; Mureithi, N.W.; Langthjem, M.A. Flow-Induced Vibrations: Classifications and Lessons from Practical Experiences, 2nd ed.; Academic Press: London, UK, 2014.

17. Hara, F. Experimental study on sloshing characteristics of a flowing liquid in a tank. J. JSME Int. J. Ser. III 1990, 33, 330-338. [CrossRef]

18. Firouz-Abadi, R.D.; Haddadpour, H.; Noorian, M.A.; Ghasemi, M. A 3D BEM model for liquid sloshing in baffled tanks. J. Int. J. Numer. Meth. Eng. 2008, 76, 1419-1433. [CrossRef]

19. Faltinsen, O.M.; Timokha, A.N. Sloshing, 1st ed.; Cambridge University Press: New York, NY, USA, 2009.

20. Saeki, S.; Madarame, H.; Okamoto, K. Self-induced sloshing excited by a horizontally injected plane jet. J. Fluid Mech. 2001, 448, 81-114. [CrossRef] 